\title{
GENERALIZED DEMATEL TECHNIQUE WITH CENTRALITY MEASUREMENTS
}

\author{
Chin-Yi CHENª, Gwo-Hshiung TZENG ${ }^{\mathrm{b}}$, Jih-Jeng HUANG ${ }^{\mathrm{c}}$ \\ ${ }^{a}$ Department of Business Administration, Chung Yuan Christian University, Chung Li, Taiwan \\ ${ }^{b}$ Graduate Institute of Urban Planning, College of Public Affairs, \\ National Taipei University, Taoyuan, Taiwan \\ ${ }^{c}$ Department of Computer Science and Management, Soochow University, Taipei, Taiwan
}

Received 02 November 2014; accepted 17 September 2015

\begin{abstract}
DEMATEL technique is a useful tool for understanding the influential relationship between criteria of a systematic problematique in structural modeling, and has received much attention in the field of decision analysis recently. However, the past papers focused on the applications of DEMATEL technique and ignored the convergence problem of the approach. In addition, two simple indicators, i.e., in-degree and out-degree centralities, used in DEMATEL technique cannot fully represent the insight of the network relationship. In this paper, we propose a general DEMATEL technique which incorporated the concept based on interaction diminishing effect. The traditional DEMATEL technique can be considered as a special case of the proposed method when we ignore the effect. Later, we give six important indicators which can be used in DEMATEL technique to conclude the relative importance of criteria. In addition, a numerical example is used to demonstrate the proposed method and the applications of the indicators.
\end{abstract}

Keywords: decision analysis, DEMATEL technique, influential relationship, systematic problematique, structural modeling, indicators.

JEL classification: C67.

\section{Introduction}

Structural modeling is still an attractive and visual tool for exploring the influential relationship between criteria (or called aspects, factors, attributes, or elements), although it has already been developed for half a century. For example, the decision making trial and evaluation laboratory (DEMATEL) technique was first developed in the 1970s (Fontela, Gabus 1974, 1976; Warfield 1976). According to the Google Scholar statistics, there are over 350 academic papers using DEMATEL in their titles since 2005, which justify the practical applications of DEMATEL.

Corresponding author Jih-Jeng Huang

E-mail: jjhuang@scu.edu.tw 
The concept of DEMATEL technique comes from the well-known Leontief's input and output model (Leontief 1949), which has been widely used in economics. The major advantage of DEMATEL technique is that it can obtain the structural model of a systematic problematique through investigating the strength of binary relations (pairwise comparison) between entries.

Compared to the past papers, this paper focuses on the issues of the convergence problem and indicator development in DEMATEL technique. The convergence problem of DEMATEL technique can be considered as the violation of Hawkins-Simon condition or strictly diagonally dominant matrices (Bidard 2007). In this situation, DEMATEL technique cannot obtain a convergent solution and, therefore, we will develop a modified DEMATEL technique to deal with this issue here. On the other hand, the conventional DEMATEL technique only uses the sums of rows and columns of total relation matrix to give decision-makers information about the relative importance of entries. In this paper, we propose six importance indicators of criteria for decision-makers to understand the insight of the problematique.

The remaining sections are organized as follows. In Section 2, the contents of DEMATEL technique are outlined. We discuss the convergence problem of DEMATEL technique in Section 3. In Section 4, a modified DEMATEL and centrality measurements of criteria are proposed. We will give a numerical example and compare the result with the conventional DEMATEL technique in Section 5. Finally, we present the discussion and conclusion in the last section.

\section{Descriptions of DEMATEL technique}

The DEMATEL technique, developed by the Science and Human Affairs Program of the Battelle Memorial Institute of Geneva between 1972 and 1976, is used for researching and solving complicated and intertwined problem groups (Fontela, Gabus 1974, 1976; Warfield 1976). DEMATEL was developed in the belief that pioneering and appropriate use of scientific research methods could improve understanding of a specific problematique, a cluster of intertwined problems, and contribute to identification of workable solutions by a hierarchical structure.

The methodology, according to the concrete characteristics of objective affairs, can confirm the interdependence between entries and restrict the relationship that reflects the characteristic with an essential system and development trend (Hori, Shimizu 1999). Using the DEMATEL method to size and process individual subjective perceptions, brief and impressionistic human insights into problem complexity can be gained. Following the DEMATEL process, the result of the analysis is a visual representation, an individual map of the mind, according to which one can organize one's own actions in the world, if one is to keep internally coherent to respect one's implicit priorities and to reach one's secret goals. Therefore, using DEMATEL technique provides an easy way for researchers to study the structure of a systematic problematique for various issues in the real world.

For example, these papers are distributed over evaluating intertwined effects in investment project (Altuntas, Dereli 2012, 2015), e-learning programs (Tzeng et al. 2007), 
customers' choice behavior model (Hsu et al. 2007), environment assessment (Tseng, Lin 2009; Chen et al. 2009, 2010), service-industry evaluation (Chen et al. 2011; Cheng et al. 2012; Liu et al. 2013), quality control (Dey et al. 2012; Tsai, Hsu 2010; Wang et al. 2014), and risk evaluation (Chang, Cheng 2011; Ou Yang et al. 2009, 2013). On the other hand, many papers have extended DEMATEL technique to consider fuzzy environments (Altuntas et al. 2014; Fekri et al. 2009; Jeng, Tzeng 2011; Lin, Wu 2004; Hsu et al. 2007; Hu et al. 2012; Lu et al. 2013; Shen, Tzeng 2014) and stochastic DEMATEL (Tamura, Akazawa 2005; Tamura et al. 2006).

The original concept of DEMATEL technique comes from Leontief's input and output model (Leontief 1949), which describes the processes of how one sector distributes its output to the other sectors of the economy (Tzeng, Tsaur 1995). The outline of the DEMATEL technique can be described as follows. First, respondents are asked to indicate the binary relation and the strength of each relation from 0 to 4 ; where measuring scale from 0 to 4 can be presented by nature language, as (0) represents no influence whatsoever, (1) represents low influence, (2) represents medium influence, (3) represents high influence, and (4) represents extremely high influence (Peng, Tzeng 2013). A higher score indicates that the respondent has expressed that factor $i$ exerts a stronger possible direct influence on the inability of factor $j$, or, in positive terms, that greater improvement of $i$ is required to improve $j$. Then, the normalized direct influence matrix $\boldsymbol{D}$ can be obtained by normalizing the direct influence matrix $\boldsymbol{X}=\left[x_{i j}\right]$, in which all principal diagonal elements are equal to zero such that

$$
\boldsymbol{D}=s \cdot \boldsymbol{X}, s>0,
$$

where

$$
s=\min _{i, j}\left\{1 / \max _{1 \leq i \leq n} \sum_{j=1}^{\mathrm{n}}\left|x_{i j}\right|, 1 / \max _{1 \leq j \leq n} \sum_{i=1}^{\mathrm{n}}\left|x_{i j}\right|\right\} .
$$

Then, the total relation matrix $\boldsymbol{F}$ can be obtained by

$$
\boldsymbol{F}=\sum_{i=1}^{\infty} \boldsymbol{D}^{i}=\boldsymbol{D}(\boldsymbol{I}-\boldsymbol{D})^{-1}
$$

where $I$ denotes the identity matrix.

[Explanation]:

$$
\begin{aligned}
& \boldsymbol{F}=\sum_{i=1}^{h} \boldsymbol{D}^{i}=\boldsymbol{D}+\boldsymbol{D}^{2}+\boldsymbol{D}^{3}+\ldots+\boldsymbol{D}^{h}=\boldsymbol{D}\left(\boldsymbol{I}+\boldsymbol{D}+\boldsymbol{D}^{2}+\ldots+\boldsymbol{D}^{h-1}\right)\left[(\boldsymbol{I}-\boldsymbol{D})(\boldsymbol{I}-\boldsymbol{D})^{-1}\right]= \\
& \boldsymbol{D}\left(\boldsymbol{I}-\boldsymbol{D}^{h}\right)(\boldsymbol{I}-\boldsymbol{D})^{-1}, \text { then, } \\
& \boldsymbol{F}=\boldsymbol{D}(\boldsymbol{I}-\boldsymbol{D})^{-1}, \text { when } \lim _{h \rightarrow \infty} \boldsymbol{D}^{h}=[0]_{n \times n},
\end{aligned}
$$

where $\mathbf{D}=\left[d_{i j}\right]_{n \times n}, 0 \leq d_{i j}<1,0<\sum_{j=1}^{n} d_{i j} \leq 1,0<\sum_{i=1}^{n} d_{i j} \leq 1$. If at least one row or column of summation is equal to 1 (but not all) in $\sum_{j=1}^{n} d_{i j}$ and $\sum_{i=1}^{n} d_{i j}$, then we can guarantee
$\lim _{h \rightarrow \infty} D^{h}=[0]_{n \times n}$. 
Furthermore, decision-makers can depict the network relationship diagram according to the result of each row and column sums. However, we ignore the succeeding steps of DEMATEL and just focus on the purpose here. Readers can refer to (Tzeng, Huang 2011) to understand the content of DEMATEL technique in detail.

Recently, many papers considered using DEMATEL to solve various decision problems. For example, Shieh et al. (2010) used DEMATEL in identifying key success factors of hospital service quality; Tzeng and Lin (2009) developed a cause and effect model of municipal solid waste management in Metro Manila by fuzzy DEMATEL; Chang and Cheng (2011) employed DEMATEL to evaluating the risk of failure; Bai and Sarkis (2013) combined grey and DEMATEL to evaluate business process management critical success factors. These papers also justify the reason of a general DEMATEL which can consider more complex decision problems.

\section{Convergence condition and problem of DEMATEL}

Since the concept of DEMATEL technique comes from Leontief's input and output analysis (I-O analysis), it shares the same conditions that the matrix $(I-D)^{-1}$ (total output vector $\boldsymbol{x}=\boldsymbol{F}(\boldsymbol{I}-\boldsymbol{D})^{-1}$ or written $\boldsymbol{x}^{\prime}=(\boldsymbol{I}-\boldsymbol{D})^{-1} \boldsymbol{F}^{\prime}$, where the superscript ' denotes the transpose operation, and $F$ denotes the final demand in I-O analysis) should be existent and positive (Tzeng, Tsaur 1995). Let us consider the following theorems and definitions to describe the issues of invariability and nonnegative inverse in DEMATEL technique.

Definition 1. If $\boldsymbol{A}$ is a real $n \times n$ matrix and can be written in the form $\boldsymbol{A}=s \boldsymbol{I}-\boldsymbol{B}$ where $\boldsymbol{B} \geq 0$ and $s>\rho(\boldsymbol{B})$, then $\boldsymbol{A}$ is called a nonsingular $M$-matrix (Frobenius 1912), where $\rho(\cdot)$ denotes the spectral radius.

Definition 2. If $\boldsymbol{A}$ is a real $n \times n$ matrix, it is said to be inverse positive if $\boldsymbol{A}^{-1} \geq 0$. If $\boldsymbol{A}^{-1}>0$, then $\boldsymbol{A}$ is said to be strictly inverse positive.

Definition 3. A real $n \times n$ matrix $\boldsymbol{A}$ is said to be reducible if there exists a permutation matrix $\boldsymbol{P}$ such that

$$
\boldsymbol{C}=\boldsymbol{P} \boldsymbol{A} \boldsymbol{P}^{\prime}=\left[\begin{array}{cc}
\boldsymbol{A}_{11} & \boldsymbol{A}_{12} \\
\mathbf{0} & \boldsymbol{A}_{22}
\end{array}\right],
$$

where $A_{11} \in R^{r \times r}, A_{22} \in R^{(n-r) \times(n-r)}$, and $A_{12} \in R^{r \times(n-r)}, \forall r \in(0, n)$.

Definition 4. A real $n \times n$ matrix $\boldsymbol{A}$ is said to be irreducible if it is not reducible.

Definition 5. A directed graph $G$ is strongly connected if for any ordered pair $(i, j)$ of vertices of $G$, there exists a sequence of edges which leads from $i$ to $j$.

Let $a_{i j}$ be the entry of a real $n \times n$ matrix $\boldsymbol{A}$. Definition 5 indicates that if there exists a sequence of $q$ edges from $i$ to $j, a_{i j}^{(q)}>0$, it translates into Theorem 1 .

Theorem 1. A real $n \times n$ matrix $\boldsymbol{A}$ is said to be irreducible iff $G(\boldsymbol{A})$ is strongly connected in graph.

Theorem 2. Let $\boldsymbol{A}$ be a real $n \times n$ nonsingular $M$-matrix. Then the following properties are equivalent: 
(i) $\boldsymbol{A}^{-1}$ is nonnegative.

(ii) The principal minors of $\boldsymbol{A}$ are strictly positive (called the Hawkins-Simon condition).

(iii) If $\boldsymbol{A}$ is irreducible, then $\boldsymbol{A}$ is strictly inverse positive.

Clearly, the matrix $(\boldsymbol{I}-\boldsymbol{D})$ in DEMATEL is a special case of an $M$-matrix, where $s=1$. From Theorem 2, it can be seen that if the matrix $(\boldsymbol{I}-\boldsymbol{D})$ in DEMATEL is a nonsingular $M$-matrix, we can ensure that the matrix $(\boldsymbol{I}-\boldsymbol{D})^{-1} \geq 0$ exists, no matter if it is reducible or irreducible. Usually, the well-known Hawkins-Simon condition is used to ensure that $(\boldsymbol{I}-\boldsymbol{D})$ is a nonsingular $M$-matrix. However, it is cumbersome in practice when dealing with complex problems, since it needs to calculate each principal minor of the matrix. Note that readers who are interested in the characteristics of the Hawkins-Simon condition can refer to Bidard (2007) for a more detailed description. In addition, if the Hawkins-Simon condition is not satisfied, we do not even know how to modify the matrix, $\boldsymbol{X}$ or $\boldsymbol{D}$, in DEMATEL. Hence, instead of considering the Hawkins-Simon condition here, we introduce the method of strictly diagonally dominant matrices as follows.

Definition 6. A real $n \times n$ matrix, $\boldsymbol{A}$, is a strictly diagonally dominant matrix if the magnitude of the diagonal element in a row/column is larger than the sum of the magnitudes of all the other (non-diagonal) entries in that row/column.

Theorem 3. The Hawkins-Simon condition is equivalent to the condition of strictly diagonally dominant matrices.

Theorem 3 claims that if the matrix $(I-D)$ is diagonally dominant, that is,

$$
\sum_{j \neq i}\left|a_{i j}\right|<\left|a_{i i}\right|, i=1, \ldots, n \text { or } \sum_{i \neq j}\left|a_{i j}\right|<\left|a_{j j}\right|, j=1, \ldots, n
$$

or, if the matrix $(\boldsymbol{I}-\boldsymbol{D})$ is irreducible and

$$
\sum_{j \neq i}\left|a_{i j}\right| \leq\left|a_{i i}\right|, i=1, \ldots, n \text { or } \sum_{i \neq j}\left|a_{i j}\right| \leq\left|a_{j j}\right|, j=1, \ldots, n .
$$

With strict inequality for at least one $i$ or $j$, then $(I-D)^{-1}$ can be ensured to be positive and existent.

Proof. Let $\boldsymbol{A}$ be diagonally dominant, the diagonal matrix of $\boldsymbol{A}$ is represented as $\operatorname{diag}\left[a_{11}, \ldots, a_{n n}\right]$ and define $\boldsymbol{B}=\boldsymbol{I}-\boldsymbol{D}^{-1} \boldsymbol{A}$. Then, if $\boldsymbol{A}$ is a diagonally dominant matrix, it implies that

and

$$
\sum_{j \neq i}\left|b_{i j}\right|<1, i=1, \ldots, n
$$

$$
\rho(B)<1,
$$

according to Gershgorin's circle theorem (Gershgorin 1931).

Since $\boldsymbol{D}^{-1} \boldsymbol{A}=\boldsymbol{I}-\boldsymbol{B}$ and $\rho(\boldsymbol{B})<1, \boldsymbol{D}^{-1} \boldsymbol{A}$ is an $M$-matrix, consequently $\boldsymbol{A}$ is an $M$ matrix.

Definition 7. If $\boldsymbol{A}$ is a real $n \times n$ matrix and can be written in the form $\boldsymbol{A}=s \boldsymbol{I}-\boldsymbol{B}$ where $\boldsymbol{B} \geq 0$ and $s=\rho(\boldsymbol{B})$, then $\boldsymbol{A}$ is a singular matrix. 
According to the above definitions, we can conclude that the convergence problem of DEMATEL can only happen when the normalized direct relation matrix is a stochastic matrix, i.e., $\rho(B)=1$, if the self-loop effect is ignored. In this situation, $(\boldsymbol{I}-\boldsymbol{D})^{-1}$ will go to infinity and result in the convergence problem. To overcome the above problem, we can develop a modified DEMATEL technique to obtain the approximate solution as the following section.

\section{Modified DEMATEL and centrality measurements}

In this paper, we consider to extend DEMATEL technique to be workable in any situations. Lee et al. (2013) provided a revised DEMATEL technique to solve the convergence problem by replacing the normalizing parameter $s$ as:

$$
s=\min _{i, j}\left\{1 / \max _{1 \leq i \leq n} \sum_{j=1}^{\mathrm{n}}\left|x_{i j}\right|, 1 /\left(\varepsilon+\max _{1 \leq j \leq n} \sum_{i=1}^{\mathrm{n}}\left|x_{i j}\right|\right)\right\},
$$

where $\varepsilon$ denotes a very small positive number. Then, other steps are similar to the traditional DEMATEL technique. Note that Eq. (9) was proposed by Lee et al. (2013) to solve the convergence problem of DEMATEL. It is an approximate formula of Eq. (2). However, the proposed method can ensure the convergence of DEMATEL. Hence, we use Eq. (2) here to normalize the direct influence matrix. In contrast to the above method, the proposed method can derive a closed-form solution and provides more flexible way to apply DEMATEL technique in more complex situations.

The main change of the proposed method is the process of calculating total relation matrix. Here, $\boldsymbol{D}$ is normalized direct relation matrix and can be viewed as the first-order weighted interaction matrix. The second and other high-order weighted interaction matrix can calculate its corresponding indirect relation matrix $D^{2}, D^{3}, \ldots$ and so on. In addition, we should also consider the interaction diminishing effect. Note that the interaction diminishing effect can be considered as the control parameter of DEMATEL technique to weight the level of interaction effect. The larger of the interaction diminishing effect, the larger the influence of the interaction effect.

Hence, the total relation matrix, $F$, here can be calculated by summing $D,(1-\alpha) D^{2}, \ldots$ as:

$$
\begin{gathered}
\boldsymbol{F}=\lim _{h \rightarrow \infty}\left[\boldsymbol{D}+(1-\alpha) \boldsymbol{D}^{2}+\cdots+(1-\alpha)^{h-1} \boldsymbol{D}^{h}\right]=\boldsymbol{D}[\boldsymbol{I}-(1-\alpha) \boldsymbol{D}]^{-1}, \\
\text { when } \lim _{h \rightarrow \infty} \boldsymbol{D}^{h}=[0]_{h \times n},
\end{gathered}
$$

where $\alpha \in[0,1]$ denotes the interaction diminishing effect which determines the level of the decreasing velocity of the interaction effect.

Note that when $\alpha=0$, Eq. (8) reduces to the original DEMATEL. On the other hand, when $\alpha=1$, only the direct relation matrix is considered. The degree of alpha indicates the importance of the interaction effect between criteria. In this paper, we adopt $\alpha=0.2$ to deal with the interaction effect for only the reason of demonstration. However, a decision-maker can determine an appropriate alpha value according to the strength of the interaction ef- 
fect. For example, if we consider to calculate the maximum interaction effect, we can set $\alpha=0$; the medium interaction effect, we can set $\alpha=0.5$; the strong interaction, we can set $\alpha=0.2$, and so on.

The convergence problem of DEMATEL technique happens when $(\boldsymbol{I}-\boldsymbol{D})^{-1}$ is singular and $\alpha=0$, according to Eq. (10). Hence, a simple way to solve the above problem is to set $\alpha$ as a small positive number to obtain an approximate solution of DEMATEL. In addition, the result of the proposed model is convergent iff $\mathbf{Z}=\Lambda[\boldsymbol{I}-(1-\alpha) \boldsymbol{\Lambda}]^{-1}, \forall \alpha \in(0,1]$ in generalized DEMATEL technique.

Proof:

\section{Case 1. If $\Lambda$ is a nonsingular matrix}

The determinant of $(1-\alpha) \Lambda, \forall \alpha \in[0,1]$ is less than 1 . Hence, the determinant of $[\boldsymbol{I}-(1-\alpha) \boldsymbol{\Lambda}], \forall \alpha \in[0,1]$, is larger than zero and ensures $\mathbf{Z}=\boldsymbol{\Lambda}[\boldsymbol{I}-(1-\alpha) \boldsymbol{\Lambda}]^{-1}=\boldsymbol{C}$, where $C$ is a constant matrix.

\section{Case 2. If $\Lambda$ is a singular matrix}

Since $\Lambda$ is a singular matrix, the determinant of $(1-\alpha) \Lambda, \forall \alpha \in[0,1]$ is less than 1 . Hence, the determinant of $\Lambda[I-(1-\alpha) \Lambda], \forall \alpha \in[0,1]$, is larger than zero and ensures $\mathbf{Z}=\boldsymbol{\Lambda}[\boldsymbol{I}-(1-\alpha) \boldsymbol{\Lambda}]^{-1}=\boldsymbol{C}$, where $C$ is a constant matrix.

The above results show the convergence problem of DEMATEL can be solved by the proposed method iff $\alpha \in(0,1]$.

On the other hand, in traditional DEMATEL technique, the sums of rows and columns of total relation matrix are the only two indicators to understand the relative importance/ influence of entities in a problematique. Here, we provide the following indicators for decision-makers to increase the insight of the network.

Definition 8. A network can be represented by a graph $(\boldsymbol{N}, \boldsymbol{G})$, which consists of a set of nodes $N=\{1, \ldots, n\}$ and an $n \times n$ matrix $\boldsymbol{G}=\left[g_{i j}\right], i, j \in N$ (referred to as an adjacency matrix), where $g_{i j} \in\{0,1\}$ represents the availability of an edge from node $i$ to node $j$.

1. In-degree and out-degree centralities: in-degree is the number of incoming edges, i.e., the column sums of the total relation matrix, and out-degree is the number of outgoing ones, i.e., the row sums of the total relation matrix. Hence, node $i$ 's in-degree and out-degree centralities can be respectively defined as

$$
C_{i d}\left(n_{i}\right)=\frac{\sum_{j} \omega_{j i} g_{j i}}{\omega^{*}|N|},
$$

and

$$
C_{o d}\left(n_{i}\right)=\frac{\sum_{j} \omega_{i j} g_{i j}}{\omega^{*}|N|},
$$

where $\omega_{i j}$ denotes the link weights, i.e., the $i$ th row and $j$ th column of the total relation matrix, $\omega^{*}$ is the maximum value of all link values in the network and $|N|$ denotes the maximum linked number of the network.

2. O-I index: $O-I$ index measures the net influence of a node in the network. The outdegree of Node $i$ indicates the influence of Node $i$ to all other nodes; the in-degree 
of Node $i$ indicates the influence of Node $i$ from all other nodes. Hence, $O-I$ index of Node $i$ is defined as:

$$
O-I \operatorname{index}\left(n_{i}\right)=\frac{\left(C_{o d}\left(n_{i}\right)-C_{i d}\left(n_{i}\right)\right)}{\left(C_{i d}\left(n_{i}\right)+C_{o d}\left(n_{i}\right)\right)} \in[0,1] .
$$

3. Closeness centrality: closeness is based on the inverse of the distance of each node to every other node in the network. A node is probably considered important if it is relatively close to all other nodes. The closeness centrality can be defined by:

$$
C_{c}\left(n_{i}\right)=\frac{\omega^{*}(|N|-1)}{\sum_{j \neq i} \omega_{i j} d(i, j)},
$$

where $d(i, j)$ denotes distance between nodes $i$ and $j$.

4. Betweenness centrality: betweenness measures the degree to which a given node lies on the shortest paths (geodesics) between other nodes in the network. That is, it counts the number of shortest paths between $i$ and $k$ that node $j$ resides on. High betweenness nodes connect different groups and have control over the flow of information in a network. The betweenness centrality can be defined as:

$$
C_{b}\left(n_{i}\right)=\frac{\sum_{j<k} \omega_{i j} g_{j k}\left(n_{i}\right) / g_{j k}}{\omega^{*}\left(|N|^{2}+|N|+2\right)},
$$

where $g_{j k}$ denotes the number of geodesics connecting $j k$, and $g_{j k}\left(n_{i}\right)$ is the number that node $i$ is on. The denominator of Eq. (15) indicates all possible combinations of two nodes excluding the center of the stars (Freeman 1977, 1979).

5. Eigenvector centrality: Eigenvector centrality is often connected with the notion of power or the idea that a node is important if it is connected to other important nodes. While other centralities reward all links equally, eigenvector centrality makes differences by including also the links of the neighbors and of the neighbors of the neighbors etc. The eigenvector centrality of node $i$ can be defined as:

$$
\begin{gathered}
C_{e}\left(n_{i}\right)=\frac{c_{i}}{\sqrt{0.5}}, \\
\lambda c_{e}=\boldsymbol{W} \boldsymbol{c}_{e}, \\
\boldsymbol{c}_{e}=\left[c_{1}, \ldots, c_{n}\right]^{\prime},
\end{gathered}
$$

where $\lambda$ is the largest eigenvalue of the adjacency matrix $W$ and $c_{e}$ is the corresponding eigenvector. Independently from the network size, the maximum value of denominator of Eq. (16) is always $\sqrt{0.5}$ (Bonacich 1972).

Next, we give a numerical example to demonstrate the proposed method and further explanation for the problematique according to the proposed indicators.

\section{A numerical example}

Quality control is a process by which entities review the quality of all factors involved in production. In project management, quality control requires the project manager and the 
project team to inspect the accomplished work to ensure its alignment with the project scope. In this example, six critical factors $\left(S_{1}\right.$ to $\left.S_{6}\right)$ are considered in the project management, such as: (1) agreeing on setting the project goals, (2) developing clearly defined plans with assigned responsibilities and accountabilities, (3) managing the project scope effectively, (4) cultivating constant effective communications, (5) making sure you have management support, (6) expanding the competence sets for enhancing the ability in implementation. The decision-maker wants to understand the relationship and relative importance between critical factors to appropriately allocate and distribute resource.

Let the initial direct influence matrix be quantified by an expert as:

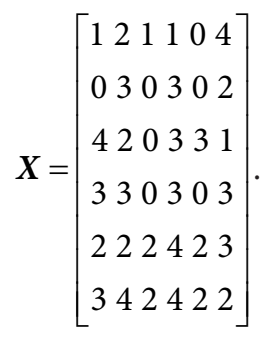

First, we normalize the above direct-influence matrix as:

$$
\boldsymbol{D}=\left[\begin{array}{llllll}
0.0556 & 0.1111 & 0.0556 & 0.0556 & 0.0000 & 0.2222 \\
0.0000 & 0.1667 & 0.0000 & 0.1667 & 0.0000 & 0.1111 \\
0.2222 & 0.1111 & 0.0000 & 0.1667 & 0.1667 & 0.0556 \\
0.1667 & 0.1667 & 0.0000 & 0.1667 & 0.0000 & 0.1667 \\
0.1111 & 0.1111 & 0.1111 & 0.2222 & 0.1111 & 0.1667 \\
0.1667 & 0.2222 & 0.1111 & 0.2222 & 0.1111 & 0.1111
\end{array}\right] .
$$

Here, we use the proposed method and respectively let $\alpha=0, \alpha=0.1, \alpha=0.2$, and $\alpha=$ 0.3 here to calculate the full direct/indirect influence matrix $\boldsymbol{F}$ of DEMATEL as:

$$
\begin{aligned}
\boldsymbol{F}_{0} & =\left[\begin{array}{llllll}
0.2308 & 0.3713 & 0.1255 & 0.3186 & 0.0781 & 0.4363 \\
0.1346 & 0.3839 & 0.0446 & 0.3838 & 0.0446 & 0.2898 \\
0.4423 & 0.4394 & 0.0943 & 0.5036 & 0.2521 & 0.3756 \\
0.3654 & 0.4822 & 0.0777 & 0.4740 & 0.0703 & 0.4460 \\
0.4038 & 0.5178 & 0.2184 & 0.6445 & 0.2324 & 0.5312 \\
0.4615 & 0.6558 & 0.2182 & 0.6677 & 0.2289 & 0.5041
\end{array}\right] ; \\
\boldsymbol{F}_{0.1} & =\left[\begin{array}{llllll}
0.1881 & 0.3074 & 0.1092 & 0.2539 & 0.0606 & 0.3813 \\
0.1016 & 0.3321 & 0.0329 & 0.3320 & 0.0328 & 0.2455 \\
0.3869 & 0.3564 & 0.0724 & 0.4192 & 0.2305 & 0.2996 \\
0.3159 & 0.4056 & 0.0589 & 0.3979 & 0.0519 & 0.3787 \\
0.3337 & 0.4155 & 0.1923 & 0.5400 & 0.2044 & 0.4403 \\
0.3894 & 0.5478 & 0.1914 & 0.5580 & 0.2007 & 0.4079
\end{array}\right] ;
\end{aligned}
$$


and

$$
\boldsymbol{F}_{0.2}=\left[\begin{array}{llllll}
0.1573 & 0.2613 & 0.0972 & 0.2073 & 0.0476 & 0.3421 \\
0.0777 & 0.2944 & 0.0246 & 0.2942 & 0.0244 & 0.2137 \\
0.3472 & 0.2969 & 0.0563 & 0.3587 & 0.2150 & 0.2444 \\
0.2804 & 0.3501 & 0.0452 & 0.3431 & 0.0388 & 0.3299 \\
0.2828 & 0.3423 & 0.1734 & 0.4649 & 0.1839 & 0.3749 \\
0.3373 & 0.4703 & 0.1722 & 0.4789 & 0.1802 & 0.3385
\end{array}\right] ;
$$

$$
\boldsymbol{F}_{0.3}=\left[\begin{array}{llllll}
0.1339 & 0.2265 & 0.0880 & 0.1720 & 0.0375 & 0.3129 \\
0.0597 & 0.2656 & 0.0185 & 0.2655 & 0.0183 & 0.1896 \\
0.3173 & 0.2525 & 0.0439 & 0.3132 & 0.2033 & 0.2023 \\
0.2536 & 0.3081 & 0.0349 & 0.3018 & 0.0291 & 0.2928 \\
0.2440 & 0.2874 & 0.1590 & 0.4082 & 0.1681 & 0.3257 \\
0.2979 & 0.4119 & 0.1577 & 0.4193 & 0.1646 & 0.2860
\end{array}\right]
$$

The degree of alpha indicates the importance of the interaction effect between criteria. In this paper, we adopt $\alpha=0.2$ to deal with the interaction effect for only the reason of demonstration. However, a decision-maker can determine an appropriate alpha value according to the strength of the interaction effect. For example, if we consider to calculate the maximum interaction effect, we can set $\alpha=0$; the medium interaction effect, we can set $\alpha=0.5$; the strong interaction, we can set $\alpha=0.2$, and so on.

Next, we can calculate the following indicators, which are mentioned previous, of $\boldsymbol{F}_{0.2}$ to understand the insight of the network as shown in Table 1, according to Eqs. (11)-(16), respectively.

Table 1. Full direct/indirect influence matrix with various alpha

\begin{tabular}{ccccccc}
\hline$F_{0.2}$ & $\begin{array}{c}\text { In-degree } \\
\text { Centrality } \\
\text { Eq. (11) }\end{array}$ & $\begin{array}{c}\text { Out-degree } \\
\text { Centrality } \\
\text { Eq. (12) }\end{array}$ & $\begin{array}{c}\text { O-I index } \\
\text { Eq. (13) }\end{array}$ & $\begin{array}{c}\text { Closeness } \\
\text { Centrality } \\
\text { Eq. (14) }\end{array}$ & $\begin{array}{c}\text { Betweenness } \\
\text { Centrality } \\
\text { Eq. (15) }\end{array}$ & $\begin{array}{c}\text { Eigenvector } \\
\text { Centrality } \\
\text { Eq. }(16)\end{array}$ \\
\hline$S_{1}$ & 0.516 & 0.387 & -0.143 & 0.128 & 0.050 & 0.515 \\
\hline$S_{2}$ & 0.701 & 0.323 & -0.369 & $0.195^{\star}$ & 0.000 & 0.583 \\
\hline$S_{3}$ & 0.198 & 0.528 & $0.455^{*}$ & 0.083 & $0.200^{*}$ & 0.500 \\
\hline$S_{4}$ & $0.747^{*}$ & 0.483 & -0.215 & 0.122 & 0.000 & $0.640^{*}$ \\
\hline$S_{5}$ & 0.240 & 0.634 & 0.451 & 0.074 & 0.000 & 0.575 \\
\hline$S_{6}$ & 0.642 & $0.688^{*}$ & 0.035 & 0.074 & 0.000 & 0.636 \\
\hline
\end{tabular}

For example, the In-degree Centrality of $S_{1}$ is calculated by Eq. (11) as $(0.1573+0.0777+\ldots+0.3373) \div(\max \{0.1573,0.2613, \ldots, 0.3385\} \times 6)=0.516$.

On the other hand, the Out-degree Centrality of $S_{1}$ is calculated by Eq. (12) as $(0.1573+0.2613+\ldots+0.3421) \div(\max \{0.1573,0.2613, \ldots, 0.3385\} \times 6)=0.387$.

Other indicators can also be derived step by step using the similar way above. 
The traditional DEMATEL technique only uses the information of the sum of rows and the sum of columns of the total relation matrix to the relative influence/importance of entities. Note that the conventional DEMATEL uses unscaled in-degree and out-degree centralities and the proposed methods scale the above two indicators. That is, their results are different. In contrast, the proposed method can derive more insightful indicators to understand the problem of the network from the result of Table 1.

For example, $\mathrm{O}-\mathrm{I}$ index indicates $S_{3}$ is influence/important node since it has the most net-influence than others. In addition, $S_{3}$ is a critical intermediate factor (i.e., bridge), because many nodes communicate via it, according to betweenness centrality. Furthermore, $S_{2}$ is also considered as an important node since it is close to every node. It can be realized $S_{2}$ as the common factor. Finally, $S_{4}$ should be also considered as a critical node since it connects many important nodes based on the information of eigenvector centrality.

\section{Discussion and conclusions}

The applicability of DEMATEL is widespread ranging from industrial planning and decision making to city planning and design, regional environmental assessment, analyzing global world problematique, and so on. A weighted structural network will be given by analyzing quantitative data on the strength of binary relations on every two criteria. However, the issue of the convergence problem and indicator development seems to be ignored in the papers.

We can give an example to demonstrate the convergence problem of DEMATEL as follows. Let the initial direct influence matrix be represented as:

$$
\boldsymbol{X}=\left[\begin{array}{llllll}
0 & 0 & 4 & 2 & 0 & 4 \\
0 & 3 & 0 & 0 & 4 & 0 \\
4 & 0 & 0 & 4 & 0 & 2 \\
3 & 0 & 3 & 0 & 0 & 4 \\
0 & 4 & 0 & 0 & 3 & 0 \\
3 & 0 & 3 & 4 & 0 & 0
\end{array}\right] .
$$

Then, the normalization of the direct-influence matrix is calculated as:

$$
\boldsymbol{D}=\left[\begin{array}{cccccc}
0 & 0 & 0.4 & 0.2 & 0 & 0.4 \\
0 & 0.3 & 0 & 0 & 0.4 & 0 \\
0.4 & 0 & 0 & 0.4 & 0 & 0.2 \\
0.3 & 0 & 0.3 & 0 & 0 & 0.4 \\
0 & 0.4 & 0 & 0 & 0.3 & 0 \\
0.3 & 0 & 0.3 & 0.4 & 0 & 0
\end{array}\right] .
$$

Clearly, the above matrix violates the Hawkins-Simon condition and suffers the convergence problem. That is, $\boldsymbol{F}$ goes to infinity. In this situation, we can set $\alpha$ as a small positive number, said 0.0001 , and obtain the approximate solution of DEMATEL. 
The contribution of this paper is twofold. First, it provides a general formula for calculating the result of DEMATEL. Compare with the revised approach provided by Lee et al. (2013), the proposed method provides a closed-form expression and flexible way to deal with the interaction effect in DEMATEL. In addition, we incorporate the concept of the interaction diminishing effect into DEMATEL to calculate the total relation matrix. This property gives DEMATEL more flexibility to consider more realistic problems.

Second, this paper proposes extra indicators, including O-I index, closeness centrality, betweenness centrality, eigenvector centrality, to help decision-makers to understand the insight of the network. The traditional DEMATEL only uses unscaled in-degree and outdegree centralities to understand the relative influence/importance of criteria. However, other factors should be considered to fully judge the relative influence/importance of criteria. For example, from the perspective of closeness centrality, nodes having a high closeness centrality are nearby all other nodes and have advantages in accessing resources in a network or having a good overview of the agents in a network.

In order to compare with the conventional DEMATEL technique, we derive the indicators of DEMATEL as shown in Table 2.

Table 2. Full direct/indirect influence matrix in the conventional DEMATEL

\begin{tabular}{ccccc}
\hline $\boldsymbol{F}$ & $\begin{array}{c}\text { Column Sum } \\
(\text { In-degree })\end{array}$ & $\begin{array}{c}\text { Row Sum } \\
(\text { Out-degree })\end{array}$ & $\begin{array}{c}\text { Prominence } \\
(\text { Row Sum }+ \text { Column Sum })\end{array}$ & $\begin{array}{c}\text { Relation } \\
\text { (Row Sum - Column Sum })\end{array}$ \\
\hline$S_{1}$ & 2.038 & 1.561 & 3.599 & -0.477 \\
\hline$S_{2}$ & 2.850 & 1.281 & 4.131 & -1.569 \\
\hline$S_{3}$ & 0.779 & 2.107 & 2.886 & 1.328 \\
\hline$S_{4}$ & $2.992^{\star}$ & 1.916 & $4.908^{*}$ & -1.076 \\
\hline$S_{5}$ & 0.906 & $2.548^{*}$ & 3.454 & $1.642^{\star}$ \\
\hline$S_{6}$ & 2.133 & 2.286 & 4.419 & 0.153 \\
\hline
\end{tabular}

According to Table 2, it can be seen that the conventional DEMATEL indicates $S_{4}$ is the most important factor (based on Prominence) and $S_{5}$ is the most important net causer (based on Relation). However, if we compare the corresponding indicators in Table 1, we can find $S_{6}$ is the most important factor and $S_{3}$ is the most important net causer. Hence, we can conclude that the result of DEMATEL highly depends on the value of alpha and, therefore, enhances the importance of the proposed method.

In this paper, we propose a general DEMATEL with a closed-form solution to deal with the convergence problem in DEMATEL. The proposed method incorporates the concept of the interaction diminishing effect distinguishes the proposed method from others. It makes DEMATEL more flexible to consider realistic problems. In addition, instead of simply using out-degree and in-degree centralities in the traditional DEMATEL, we propose five extra indicators for decision-makers to understand the insight of the network. In addition, the conventional DEMATEL is a special case of the proposed method while alpha is equal to 0 . Hence, the proposed method can be considered as the generalization of DEMATEL. 
Although we extend the DEMATEL technique to consider more general situation and develop extra indicators to understand the insight of the network structure, several limitations of the proposed method are described as following. First, the proposed method ask an expert to quantify the strength of each relation. However, the realistic situation is usually subjective uncertainty. Hence, the extension of the proposed method under fuzzy environments should be considered. Furthermore, the proposed method added a new parameter, alpha, to reflect the interaction diminishing effect. However, the calibration of alpha should be carefully studied, because it may affect the final result significantly. These limitations can be studied further to consider more complex situations.

\section{References}

Altuntas, S.; Dereli, T. 2012. An evaluation index system for prediction of technology commercialization of investment projects, Journal of Intelligent and Fuzzy Systems 23(6): 327-343.

Altuntas, S.; Dereli, T. 2015. A novel approach based on DEMATEL method and patent citation analysis to prioritize investment projects, Expert Systems with Applications 42(3): 1003-1012. https://doi.org/10.1016/j.eswa.2014.09.018

Altuntas, S.; Selim, H.; Dereli, T. 2014. A fuzzy DEMATEL-based solution approach for facility layout problem: a case study, The International Journal of Advanced Manufacturing Technology 73(5): 749-771. https://doi.org/10.1007/s00170-014-5826-3

Bai, C.; Sarkis, J. 2013. A grey-based DEMATEL model for evaluating business process management critical success factors, International Journal of Production Economics 146(1): 281-292. https://doi.org/10.1016/j.ijpe.2013.07.011

Bidard, C. 2007. The weak Hawkins-Simon condition, Electronic Journal of Linear 16(1): 44-59. https://doi.org/10.13001/1081-3810.1181

Bonacich, P. 1972. Factoring and weighting approaches to status scores and clique identification, Journal of Mathematical Sociology 2(1): 113-120. https://doi.org/10.1080/0022250X.1972.9989806

Chang, K. H.; Cheng, C. H. 2011. Evaluating the risk of failure using the fuzzy OWA and DEMATEL method, Journal of Intelligent Manufacturing 22(2): 113-129. https://doi.org/10.1007/s10845-009-0266-x

Chen, F. H.; Hsu, T. S.; Tzeng, G. H. 2011. A balanced scorecard approach to establish a performance evaluation and relationship model for hot spring hotels based on a hybrid MCDM model combining DEMATEL and ANP, International Journal of Hospitality Management 30(4): 908-932. https://doi.org/10.1016/j.ijhm.2011.02.001

Chen, Y. C.; Lien, H. P.; Tzeng, G. H.; Yang, L. S. 2009. Combined DEMATEL technique with VIKOR method for improving environment-watershed plan strategy, in EURO Conference, 5-8 July 2009, Bonn, p. TA-37.

Chen, Z.; Xie, X. B.; Yan, L.; Zheng, Z. 2010. Analysis of external environmental influencing factors on the evolution of resource-based industrial system based on DEMATEL method, in IEEE $2^{\text {nd }}$ International Conference on Information Science and Engineering (ICISE), 04-06 December 2010, Hangzhou, China, 93-96.

Cheng, C. C.; Chen, C. T.; Hsu, F. S.; Hu, H. Y. 2012. Enhancing service quality improvement strategies of fine-dining restaurants: new insights from integrating a two-phase decision-making model of IPGA and DEMATEL analysis, International Journal of Hospitality Management 31(4): 1155-1166. https://doi.org/10.1016/j.ijhm.2012.02.003

Dey, S.; Kumar, A.; Ray, A.; Pradhan, B. B. 2012. Supplier selection: integrated theory using DEMATEL and quality function deployment methodology, Procedia Engineering 38(11): 3560-3565.

https://doi.org/10.1016/j.proeng.2012.06.411 
Fekri, R.; Aliahmadi, A.; Fathian, M. 2009. Identifying the cause and effect factors of agile NPD process with fuzzy DEMATEL method: the case of Iranian companies, Journal of Intelligent Manufacturing 20(6): 637-648. https://doi.org/10.1007/s10845-008-0153-x

Fontela, E.; Gabus, A. 1974. DEMATEL, Innovative Methods. Report No. 2, Structural analysis of the world problematique. Battelle Geneva Research Institute (Battelle Memorial Institute).

Fontela, E.; Gabus, A. 1976. The DEMATEL Observer. Institute, Geneva Research Center.

Freeman, L. C. 1977. A set of measures of centrality based on betweenness, Sociometry 40(1): 35-41. https://doi.org/10.2307/3033543

Freeman, L. C. 1979. Centrality in social networks conceptual clarification, Social Networks 1(3): 215239. https://doi.org/10.1016/0378-8733(78)90021-7

Frobenius, G. 1912. Über Matrizen aus nicht negativen Elementen. Königliche Akademie der Wissenschaften, 456-477.

Gershgorin, S. A. 1931. Uber die abgrenzung der eigenwerte einer matrix, Известия Российской академии наук, Серия математическая 6: 749-754.

Hori, S.; Shimizu, Y. 1999. Designing methods of human interface for supervisory control systems, Control Engineering Practice 7(11): 1413-1419. https://doi.org/10.1016/S0967-0661(99)00112-4

Hsu, C. Y.; Chen K. T.; Tzeng, G. H. 2007. FMCDM with fuzzy DEMATEL approach for customers' choice behavior model, International Journal of Fuzzy Systems 9(4): 236-246.

Hu, S. K.; Chuang, Y. C.; Yeh, Y. F.; Tzeng, G. H. 2012. Combining hybrid MADM with fuzzy integral for exploring the smart phone improvement in m-generation, International Journal of Fuzzy Systems 14(2): 204-214.

Jeng, D. J. F.; Tzeng, G. H. 2011. Social influence on the use of clinical decision support systems: revisiting the unified theory of acceptance and use of technology by the fuzzy DEMATEL technique, Computers and Industrial Engineering 62(3): 819-828. https://doi.org/10.1016/j.cie.2011.12.016

Lee, H. S.; Tzeng, G. H.; Yeih, W.; Wang, Y. J.; Yang, S. C. 2013. Revised DEMATEL: resolving the infeasibility of DEMATEL, Applied Mathematical Modelling 37(10-11): 6746-6757. https://doi.org/10.1016/j.apm.2013.01.016

Leontief, W. 1949. Recent developments in the study of interindustrial relationships, The American Economic Review 39(3): 211-225.

Lin, C. J.; Wu, W. W. 2004. A fuzzy extension of the DEMATEL method for group decision-making, European Journal of Operational Research 156(3): 445-455.

Liu, C. H.; Tzeng, G. H.; Lee, M. H. 2013. Strategies for improving cruise product sales using hybrid "multiple criteria decision making" models, The Service Industries Journal 33(5): 542-563. https://doi.org/10.1080/02642069.2011.614342

Lu, M. T.; Lin, S. W.; Tzeng, G. H. 2013. Improving RFID adoption in Taiwan's healthcare industry based on a DEMATEL technique with a hybrid MCDM model, Decision Support Systems 56: 259-269. https://doi.org/10.1016/j.dss.2013.06.006

Ou Yang, Y. P.; Shieh, H. M.; Tzeng, G. H. 2009. A VIKOR-based multiple criteria decision method for improving information security risk, International Journal of Information Technology and Decision Making 8(2): 267-287. https://doi.org/10.1142/S0219622009003375

Ou Yang, Y. P.; Shieh, H. M.; Tzeng, G. H. 2013. A VIKOR technique based on DEMATEL and ANP for information security risk control assessment, Information Sciences 232(5): 482-500. https://doi.org/10.1016/j.ins.2011.09.012

Peng, K. H.; Tzeng, G. H. 2013. A hybrid dynamic MADM model for problem-improvement in economics and business, Technological and Economic Development of Economy 19(4): 638-660. https://doi.org/10.3846/20294913.2013.837114

Shen, K. Y.; Tzeng, G. H. 2014. DRSA-based neuro-fuzzy inference systems for the financial performance prediction of commercial banks, International Journal of Fuzzy Systems 16(2): 173-183. 
Shieh, J. I.; Wu, H. H.; Huang, K. K. 2010. A DEMATEL method in identifying key success factors of hospital service quality, Knowledge-Based Systems 23(3): 277-282.

https://doi.org/10.1016/j.knosys.2010.01.013

Tamura, H.; Akazawa, K. 2005. Stochastic DEMATEL for structural modeling of a complex problematique for realizing safe, secure and reliable society, Journal of Telecommunications and Information Technology 4(2): 139-146.

Tamura, H.; Okanishi, H.; Akazawa, K. 2006. Decision support for extracting and dissolving consumers' uneasiness over foods using stochastic DEMATEL, Journal of Telecommunications and Information Technology 4(1): 91-95.

Tsai, W. H.; Hsu, W. 2010. A novel hybrid model based on DEMATEL and ANP for selecting cost of quality model development, Total Quality Management 2(4): 439-456. https://doi.org/10.1080/14783361003606852

Tseng, M. L.; Lin, Y. H. 2009. Application of fuzzy DEMATEL to develop a cause and effect model of municipal solid waste management in Metro Manila, Environmental Monitoring and Assessment 158(1): 519-533. https://doi.org/10.1007/s10661-008-0601-2

Tzeng, G. H.; Chiang, C. H.; Li, C. W. 2007. Evaluating intertwined effects in e-learning programs: a novel hybrid MCDM model based on factor analysis and DEMATEL, Expert Systems with Applications 32(4): 1028-1044. https://doi.org/10.1016/j.eswa.2006.02.004

Tzeng, G. H.; Huang, J. J. 2011. Multiple Attribute Decision Making: Methods and Applications. CRC Press.

Tzeng, G. H.; Tsaur, S. H. 1995. Energy demand forecast for motor freight transportation in Taiwan: application of a dynamic interregional input-output model, Journal of Applied Input-Output Analysis 2(2): 41-53.

Wang, F. K.; Hsu, C. H.; Tzeng, G. H. 2014. Applying a hybrid MCDM model for six sigma project selection, Mathematical Problems in Engineering (2014), Article ID 730934. https://doi.org/10.1155/2014/730934

Warfield, J. N. 1976. Societal systems: planning, policy, and complexity. New York: Wiley.

Chin-Yi CHEN. She is an Assistant Professor of business administration at Chung Yuan Christian University, teaches knowledge management, market research and electronic commerce. She received her $\mathrm{PhD}$ of management of technology in National Chiao Tung University. Her research interests are marketing and behavioural economics.

Gwo-Hshiung TZENG. He is a Distinguished Chair Professor at National Taipei University. He is Editor-in-Chief of the International Journal of Operations Research. He received a PhD in management science from Osaka University. His research interests include statistics, multivariate analysis, networks, routing and scheduling, multiple criteria decision making, fuzzy theory, hierarchical structure analysis for application to technology management, energy, environment, transportation systems, transportation investment, logistics, location, urban planning, tourism, technology management, electronic commerce, and global supply chains.

Jih-Jeng HUANG. PhD is an Associate Professor of computer science and information management at Soochow University, where he teaches research methods, multivariate analysis, and data mining. He received a PhD in information management from the National Taiwan University. His research interests include multiple criteria decision making, knowledge management, behavioural economics and finance, and data analysis. His work has been widely published in journals and conference proceedings for over 30 papers. 